\title{
Satzmodus und explizite Performativität von Satzäußerungen1
}

\section{Summary}

This paper critically examines some recent performativist conceptions of analysing and describing interpretation of explicit performatives. In an alternative constativist approach the paper tries to determine the illocutionary point of explicit performatives on the basis of sentence mood, lexical information (i.e. the meaning of the speech act verb), utterance meaning, speaker's authority, and some general principles of social behaviour.

\section{Das Problem: die Ableitung der illokutiven Funktion explizit performativer Äußerungen}

Mit Verwendungen von Sätzen in der Kommunikation verfolgen diejenigen, die die Sätze verwenden, nachweislich bestimmte Ziele. Eines dieser Ziele ist, beim Adressaten der Satzverwendung ein u. U. praktisches Verhalten auszulösen. In der Theorie, die sich mit diesem Aspekt von Sprache befaßt, wird ein Zusammenhang zwischen formaler Struktur der Sätze - dabei besonders außerlexikalischer Aspekte der Sätze - und Ziel der Äußerungen der Sätze in der interpersonellen Kommunikation unterstellt.2 So leitet man aus empirischen Beobachtungen die Annahme ab, daß Sätze wie

(1) (a) Tut er was?

(b) Was tut er?

Fragen ausdrücken und daß mit der Äußerung solcher Sätze gegenüber einem Adressaten das Ziel verfolgt werden kann, vom Adressaten eine Antwort auf diese Fragen zu erhalten. Entsprechend kann man für Äußerungen von Sätzen wie

(2) Er tut was. (mit fallender Intonation geäußert)

die Annahme ableiten, daß ein Sprecher mit ihnen eine „Mitteilung“ an einen Adressaten machen kann, d. h. das Ziel verfolgen kann, daß der Äußerungsadressat davon überzeugt ist, daß der vom Satz bezeichnete, im Äußerungskontext relevante Sachverhalt ${ }^{3}$ eine Tatsache - ein Faktum - ist. ${ }^{4}$ Für Äußerungen von Sätzen wie

(3) Tu was!

1 Der folgende Aufsatz ist die erweiterte Vorlage für einen Vortrag, den ich am 8. 11. 1990 an der Westfälischen Wilhelms-Universität Münster gehalten habe.

2 Siehe Recanati (1987: 15ff.), Motsch/Pasch (1987), Falkenberg (1989), Searle (1989).

3 Unter dem ,,vom Satz bezeichneten relevanten Sachverhalt" $\left(\mathrm{rSV}_{\mathrm{S}}\right)$ verstehe ich denjenigen vom Satz bezeichneten Sachverhalt, der im Äußerungskontext des Satzes direkt kommentiert werden kann. Ein „direkter“ Kommentar - siehe hierzu POSNER (1980) - ist ein Kommentar, in dem das zu Kommentierende (bzw. das, zu dem ein Kommentar gegeben wird) durch das Pronomen das oder dies bezeichnet werden kann. Siehe im übrigen PASCH (1990: 50ff.).

4 Siehe auch MOTSCH/PASCH (1984), (1987), ROLF (1986: 480). 
kann man die Annahme ableiten, daß ihr Sprecher mit ihnen das Ziel verfolgen kann, daß ihr Adressat den vom Satz bezeichneten, im Äußerungskontext relevanten Sachverhalt verwirklicht, zu einem Faktum macht. Unsere Erfahrungen mit der Verwendung solcher Ausdrücke zeigen uns, daß man die genannten Ziele auch mit Erfolg verfolgen kann. Solche Ziele stellen im wesentlichen das dar, was in der Literatur unter den Termini ,illokutive (/illokutionäre) Funktion“, ,illokutionärer Witz“ behandelt wird. Bei MoTSCH/ PASCH (1987: 27) wird die illokutive Funktion „fundamentales Ziel“ genannt. Das fundamentale Ziel ist das letzte in einer Reihe von Zielen einer sprachlichen Handlung, die mit dieser Handlung ausschlieBlich kraft der Form des Ausdrucks mit Aussicht auf Erfolg verfolgt werden können. Die dem fundamentalen Ziel vorgeordneten Ziele müssen erreicht sein, damit das fundamentale Ziel erreicht werden kann. ${ }^{5}$

Die Beispiele (1) bis (2) zeigen, daß bereits Unterschiede in der Wortstellung der Sätze ausreichen, um die möglichen illokutiven Funktionen der Äußerungen der Sätze zu differenzieren, einzuschränken. Um den Ausdruck eines Urteils, wie es in (2) illustriert wird, mit der für seine Äußerungen ableitbaren illokutiven Funktion der Mitteilung von dem einer Frage abzugrenzen, reicht es, das nichtimperativische finite Verb in die sog. Zweitposition zu bringen, das Vorfeld mit einer Nicht-w-Konstituente zu besetzen und den Satz mit fallender Intonation zu äußern (siehe (2)), d. h. einen „Deklarativsatz“ zu bilden.

Wenn diese Struktureigenschaften eine hinreichende Bedingung dafür sein sollen, daß eine Äußerung eines Satzes, der diese Eigenschaften aufweist, eine Mitteilung im oben gen. Sinne ist, müßten Sätze wie (4) (a) und (b) Ausdrücke für Urteile und ihre Äußerungen Urteilsäußerungen bzw. Behauptungen sein, und deren illokutive Funktion müßte die einer Mitteilung sein können.

(4) (a) Ich bitte Sie hiermit höflichst, diesen Antrag wohlwollend zu prüfen.

(b) Ich verspreche dir hiermit feierlich, daß ich deinen Aufsatz lesen werde.

Sätze wie die unter (4) aufgeführten nenne ich im folgenden „EPF-Sätze“. Dabei steht „EPF“ für ,explizit performative Formel“. Unter einer solchen Formel wird in der Literatur eine Einheit aus einem eine sprachliche Handlung bezeichnenden Verb im Indikativ Aktiv Präsens und seinem Subjekt in der ersten Person Singular verstanden. ${ }^{6}$ Ich will den Begriff der EPF hier aber noch dahingehend einschränken, daß ich als finites Verb einer EPF nur solche Verben zulasse, die einen Satz oder einen zu-Infinitiv als Komplement haben. Das heißt, ich will im folgenden Sätze wie Ich taufe dieses Schiff auf den Namen Maria. oder Hiermit eröffne ich die Sitzung. aus meinen Betrachtungen ausschließen.

EPF-Sätze können in der Weise verwendet werden, daß der von der EPF und ihrem Komplement beschriebene Sachverhalt ein Faktum in der Seinswelt des Referenten des Subjekts der EPF wird. Das heißt, die Satzverwendung ist für die Faktizität des vom Satz bezeichneten relevanten Sachverhalts $-\mathrm{rSV}_{\mathbf{s}}-$, konstitutiv“. Sie konstituiert diesen Sachverhalt als Faktum. ${ }^{7}$ Diesen Tatbestand habe ich im Auge, wenn ich im folgenden von „explizit performativen Äußerungen von EPF-Sätzen“ spreche. In dieser Verwendungsmöglichkeit unterscheiden sich EPF-Sätze grundlegend von Deklarativsätzen ohne EPF. Damit die EPF-Sätze auch wirklich explizit performativ verwendet werden können, müssen sie die oben für Deklarativsätze genannte typische Form haben. Ferner darf die

5 Näheres zur Zielhierarchie siehe bei MotsCH/PASCH (1987).

6 Siehe AustIN (1962: 56).

7 Siehe hierzu insbesondere STAMPE (1975: 29) und REHBOCK (1989). 
Geltung der EPF nicht durch Adverbiale (wie nicht, hoffentlich, wahrscheinlich) aufgehoben bzw. beschränkt sein, und das Komplement der EPF muß den Hauptakzent des EPFSatzes tragen.

Nun wird von manchen Sprachtheoretikern jedoch abgelehnt, die explizit performativen Äußerungen solcher Sätze als Urteilsäußerungen bzw. Behauptungen und die geäußerten Sätze als wahrheitswertfähig, ,konstativ“ bzw. ,assertiv“ zu akzeptieren, was sie sein müßten, wenn sie Ausdrücke von Urteilen wären. ${ }^{8}$ Es wird diesen Äußerungen auch nicht - und zwar konsequenterweise - die illokutive Funktion der Mitteilung zugeschrieben, sondern nur diejenige illokutive Funktion, die die vom Verb der EPF beschriebene sprachliche Handlung hat ${ }^{9}$ (in (4) sind dies die illokutive Funktion einer Bitte und die eines Versprechens). Die Annahme, daß die Äußerung des EPF-Satzes die betreffende illokutive Funktion hat, ist gerechtfertigt. Wenn es nämlich legitim ist, z. B. für die Äußerung eines Satzes wie

(5) Bitte prüfen Sie diesen Antrag wohlwollend!

als Ziel die Realisierung des von (5) bezeichneten Sachverhalts, daß der Adressat den bezeichneten Antrag wohlwollend prüft, anzunehmen, so ist es auch legitim, dies als Ziel der Äußerung von (4) (a) anzunehmen, also für die Äußerung von (4) (a) das Ziel der Erfüllung der vom Satz bezeichneten und vom finiten Verb des Satzes beschriebenen Bitte abzuleiten. ${ }^{10} \mathrm{Daß}$ eine Äußerung solcher Sätze wie (4) (a) und (b) nicht als Mitteilung, aber auch nicht als Behauptung bezeichnet werden kann, sieht man daran, daß auf sie nicht mit diesen Wörtern referiert werden kann.

Damit ergibt sich für die Analyse und theoretische Beschreibung der Interpretationen von EPF-Satz-Äußerungen das Problem, daß den Äußerungen aufgrund der im wesentlichen nichtlexikalischen Formaspekte der geäußerten Sätze als ein illokutives Ziel die illokutive Funktion der Mitteilung zugeschrieben werden können müßte und aufgrund ihrer lexikalischen Spezifik diejenige illokutive Funktion, die die sprachliche Handlung hat, die von der EPF beschrieben wird. ${ }^{11}$ Das heißt, den Äußerungen solcher Sätze müßten zwei illokutive Ziele zugeordnet werden können, und zwar nicht alternativ. Dies muß nun an sich noch kein Problem darstellen, können doch Äußerungen von Deklarativsätzen, auch wenn sie keine EPF enthalten, außer der illokutiven Funktion der Mitteilungen ein weiteres illokutives Ziel haben. So kann z. B. (2) noch als Aufforderung verwendet werden: ein Sprecher kann, wenn er weiß, daß die Faktizität des $\mathrm{rSV}_{\mathrm{s}}$ von (2) dem Adressaten als Prämisse für ein bestimmtes Verhalten $\mathrm{v}_{\mathrm{i}}$ jenseits der Übernahme der Überzeugtheit von der Faktizität des $\mathrm{rSV}_{\mathrm{s}}$ von (2) dienen kann, den Adressaten mit der Äußerung von (2) zu diesem Verhalten $v_{i}$ auffordern. Mit der Äußerung von (2) in dieser Funktion wird dann ein sog. indirekter Sprechakt der Aufforderung vollzogen. (Wichtig ist bei ,indirekten“ Sprechakten, daß die Indirektheit zweifach ist: zum einen erfolgt die Interpretation des einen illokutiven Ziels nur auf der Grundlage der Interpretation eines ande-

8 Siehe Austin (1962), GREWENDORF (1979), ROSENGREN (1984), (1985), (1988), FALKenberG (1989 und 1990), LIEDTKE (1990), REHBOCK (1989), BRANDT/ROSENGREN/ZIMMERMANN (1990).

9 Siehe hierzu vor allem FALKENBERG (1990).

10 Das soll nicht heißen, daß Sätze wie (4) (a) ohne höflichst und (5) als synonym angesehen werden müssen, wie dies offensichtlich von SEARLE (1969) praktiziert wird. Siehe STAMPES (1975: 13f.) Kritik an einer solchen Annahme.

11 FALKENBERG (1989: 463) sagt, daß explizit performative Äußerungen „die Standardzuordnung von Satztyp und Handlungstyp [verletzen]“. 
ren illokutiven Ziels, zum anderen wird eines der $\mathrm{zu}$ interpretierenden Ziele vom Satz überhaupt nicht indiziert.)

Im Falle der EPF-Satz-Äußerungen wird in der sprachphilosophischen und linguistischen Literatur seit AUSTIN (1962) ein bisher nicht beendeter Streit darüber geführt, ob den explizit performativen Äußerungen in der gleichen Weise wie den indirekten Sprechakten zwei illokutive Ziele zugeschrieben werden müssen, von denen die Realisierung des einen Vorbedingung für die Realisierung des anderen ist.

\section{Konstantivismus vs. Performativismus}

Die eine Partei in diesem Streit geht davon aus, daß der syntaktischen Form der Sätze zusammen mit den oben genannten prosodischen Phänomenen eine entscheidende Rolle bei der Ableitung der illokutiven Funktion ihrer Äußerungen zukommt. Sie analysiert alle Deklarativsätze ohne die Faktizität des $\mathrm{RSV}_{\mathrm{s}}$ einschränkende Adverbiale als Ausdrücke von Urteilen, die im Hinblick auf Wahrheit bewertet werden können. ${ }^{12}$ Diese Position nenne ich in Übereinstimmung mit einer Bezeichnung aus der englischsprachigen Literatur „Konstativismus“ (siehe STAMPE (1975): „constativism“). Die Gegenpartei spricht den explizit performativen Äußerungen die Möglichkeit ab, einen Wahrheitswert zu haben. Den Grund dafür bezieht sie - wenn auch nicht immer nachdrücklich deklariert, so doch nicht minder offensichtlich - aus der Tatsache, daß man explizit performative Äußerungen von EPF-Sätzen nicht als „Behauptungen“ bezeichnen kann und auf sie nicht mit Prädikaten wie wahr oder falsch kommentierend Bezug nehmen kann. ${ }^{13}$ Ich nenne diese Position in Übereinstimmung mit einer Bezeichnung aus der englischsprachigen Literatur „Performativismus“ (siehe STAMPE (1975): „performativist").

Nach Annahme von Konstativisten ist die Interpretation der illokutiven Funktion der von der EPF beschriebenen Handlung als illokutive Funktion der EPF-Satz-Äußerung aus der Deklarativsatzbedeutung nur inferentiell abzuleiten, ist mit dieser nicht direkt verbunden, sondern geschieht auf ,indirektem“ Wege, ${ }^{14}$ und zwar durch vorherige Ableitung einer an den Deklarativsatztyp geknüpften illokutiven Funktion der Äußerung. Nach Annahme der Performativisten kommt die Interpretation der illokutiven Funktion der explizit performativen Äußerungen von EPF-Sätzen ohne Zwischenschaltung einer Ableitung einer an den Deklarativsatztyp geknüpften illokutiven Funktion der Äußerung zustande.

Welcher der beiden Positionen ist der Vorzug zu geben? FALKENBERG (1989: 464) sagt, der Streit sei nur ,durch Abwägen der Gesamttheorien, in die beide Optionen sich einfügen“, entscheidbar, aber von einem Gesamtvergleich sei man „mangels präziser Vorschläge ... noch weit entfernt“. Ich möchte im folgenden die Position des Konstativismus in Richtung auf eine Gesamttheorie von der Interpretation sprachlicher Handlungen etwas auszubauen versuchen. Im Zusammenhang damit werde ich zu zeigen versuchen, warum ich den Konstantivismus dem Performativismus vorziehe.

12 Siehe LeWIS (1972), STAMPE (1975), BACH/HARNISH (1979), BARTSCH (1979), BIERWISCH (1979), ReCanati (1980), (1987), PASCH (1985), (1989), (1990), МOTSCH/PASCH (1987), SEARLE (1989).

13 Vgl. dazu Fußn. 8.

14 Siehe Stampe (1975), Bach/HaRnish (1979), Motsch/Pasch (1987), ReCANATI (1987). 


\section{Die Probleme des Performativismus}

Wenn man bei der Formulierung der Regeln der Interpretation der illokutiven Funktion explizit performativer Äußerungen Performativismus praktizieren will, dann muß man annehmen, daß Äußerungen von Deklarativsätzen ohne EPF die illokutive Funktion einer Mitteilung haben können und explizit performative Äußerungen von EPF-Sätzen diese Funktion nicht haben können. Damit stellt sich die Frage, woraus dieser Unterschied resultiert.

3.1. Eine Fraktion des Performativismus nimmt an, daß die Interpretation der illokutiven Funktion einer Behauptung oder Mitteilung dessen, was der EPF-Satz beschreibt, als eines der illokutiven Ziele der Äußerung blockiert wird. ${ }^{15}$ Diese Lösung ist ad hoc, denn sie ist allein durch das Bemühen diktiert, die illokutive Funktion der Mitteilung nicht als fundamentales Ziel (illokutive Funktion) der explizit performativen Äußerungen von EPF-Sätzen zuzulassen, was sie ja faktisch auch nicht ist. Ein plausibler Grund für die Annahme einer Blockierung wäre, wenn der Interpretation des Ziels der Mitteilung etwa der Faktenkontext der Äußerung entgegenstünde (siehe Ironie, Rhetorizität) oder wenn die Bedeutung eines Teilausdrucks des Satzes der Interpretation der Mitteilung des Satzinhalts selbst entgegenstünde. Ersteres ist offensichtlich nicht der Fall. Letzteres ist z. B. bei Vorkommen bestimmter Partikeln in Deklarativsätzen der Fall. So kann z. B. die Partikel bitte die Interpretation der Funktion der Mitteilung verhindern. Vgl. (6):

(6) Wir stellen jetzt bitte alle Privatgespräche ein.

In Sätzen wie (6) hat die Partikelbedeutung dasselbe Argument wie die Bedeutung des Satztyps und die mutmaßlich mit dieser korrelierte illokutive Funktion der Satzäußerung. In (6) ist dieses Argument der Sachverhalt, daß die mit wir bezeichneten Personen alle Privatgespräche einstellen. Dadurch kommt es bei den Äußerungen von Sätzen wie (6) zu einer Kollision illokutiver Funktionen, wenn man eine Korrelation von Satztypbedeutung und illokutiver Funktion annimmt, nämlich einer Kollision von „Mitteilung“ oder „Behauptung“ mit „Bitte“. Aus der Tatsache, daß die Äußerung des Satzes (6) nur als Bitte und nicht als Mitteilung oder Behauptung interpretiert werden, kann man schließen, daß für die Interpretation der illokutiven Funktion der Satzäußerung die Bedeutung der Partikel und nicht die Bedeutung des Satztyps ausschlaggebend ist. Ähn-

15 Siehe u. a. SeArLe (1969: 30), KATZ (1972: 152), BrandT/Rosengren/ZIMMERMANN (1990: 145). Andere Möglichkeiten im Rahmen des Performativismus wären: a) mit dem syntaktischen Satztyp der Deklarativsätze $\alpha$ ) keinen semantischen Satzmodus als Grundlage eines illokutiven Ziels der Satzäußerung oder $\beta$ ) keine illokutive Funktion der Satzäußerungen zu korrelieren oder b) mit EPF-Sätzen $\alpha$ ) keinen an die Deklarativsatzform gebundenen, eine illokutive Funktion der Satzäußerungen begründenden Satzmodus oder $\beta$ ) keine an die Deklarativsatzform gebundene illokutive Funktion der Satzäußerungen zu korrelieren. Alle diese Möglichkeiten wären ad-hocLösungen, weil sie nur auf die formale Verhinderung der Ableitung des illokutiven Ziels ,Mitteilung' gerichtet sind. Sie sind Ausnahmeregelungen, d. h. sie verhindern die Anwendung allgemeiner Interpretationsregeln auf die EPF-Sätze. Damit sind sie mit der Annahme einer Blockierung der Ableitung des illokutiven Ziels der Mitteilung bei explizit performativen Äußerungen von EPF-Sätzen äquivalent und lassen ihrerseits die von der Blockierungshypothese nicht beantwortete Frage offen, warum die in explizit performativen Äußerungen verwendeten Sätze Deklarativsätze sein müssen. Die Annahmen a) $\alpha$ und b) $\alpha$ lassen darüberhinaus die Frage offen, warum zwar die von EPF-Sätzen bezeichneten relevanten Sachverhalte qua Satzäußerung als Prämissen angesehen werden dürfen, die von Entscheidungsinterrogativsätzen bezeichneten relevanten Sachverhalte dagegen nicht. 
liche Beobachtungen kann man bei der Interpretation von Deklarativsätzen mit anderen Partikeln oder Satzadverbialen machen. Im Unterschied zu den Deklarativsätzen mit derartigen Partikeln oder Satzadverbialen muß man für EPF-Sätze keine Kollision zwischen Satztypbedeutung und mit ihr mutmaßlich korrelierter illokutiver Funktion der Satzäußerungen einerseits und von der EPF indizierter illokutiver Funktion andererseits annehmen. Der Grund ist, daß man annehmen kann, daß die Bedeutung der EPF zum Argument der Satztypbedeutung gehört. Dazu ist man berechtigt, weil die EPF bestimmten Manipulationen unterworfen werden kann, die charakteristischerweise an dem yorgenommen werden können, was als Argument der als Funktor aufgefaßten Bedeutung des syntaktischen Satztyps gelten kann. So zeigen Sätze wie (4) (a) und (b) Adverbiale, die die vom EPF-Verb bezeichnete sprachliche Handlung näher charakterisieren (vgl. $h o ̈ f-$ lichst, feierlich). Auch ohne diese Adverbiale wären die betreffenden Sätze noch explizit performativ verwendbar, wie sie auch explizit performativ verwendbar wären, wenn in ihnen hiermit fehlte.

Die betreffenden Adverbiale können nun auch in Sätzen auftreten, die keine EPF aufweisen. Vgl.

(7) (a) Er hat feierlich versprochen, sein Wort zu halten.

(b) Hat er feierlich versprochen, sein Wort zu halten?

In jedem Fall ist die Bedeutung des Adverbials (in (7) feierlich) mit der seines Arguments (in (7) mit der Bedeutung von er hat versprochen, sein Wort zu halten) Argument der Bedeutung des syntaktischen Satztyps (in (7) (a) des Urteils, in (7) (b) der Frage). Warum sollte sie dies nicht auch in Verwendungen von Sätzen wie (4) (a) und (b) sein können? Adverbiale wie bitte dagegen können nicht von Adverbialen wie feierlich, höflichst usw. modifiziert werden. (In Wir stellen jetzt bitte höflichst/feierlich alle Privatgespräche ein. beziehen sich höflichst, feierlich nicht auf die Bitte, sondern auf das Einstellen der Privatgespräche.)

Dieser Unterschied zwischen EPF und Adverbialen zeigt, daß es für den Aufbau des Systems möglichst genereller Regeln der Interpretation sprachlicher Ausdrücke sehr nachteilig wäre, wenn EPF dieselbe Funktion wie Satzadverbialen oder Partikeln zugeschrieben würde. Dies aber tut der Performativismus ja faktisch, wenn er Blockierungen annimmt.

3.2. Eine andere Variante des Performativismus ist es, die Art der illokutiven Funktion der Satzäußerungen allein an die Art der Äußerungsbedeutung der Satzäußerungen zu binden. Ein solcher Vorschlag wurde von REHBOCK (1989) entwickelt. REHBOCK unterscheidet Arten der Äußerungsbedeutung (,rhetischer Akte") von Deklarativsätzen in Abhängigkeit davon, ob der Sachverhalt, auf den die geäußerten Sätze referieren, unabhängig von der Äußerung des jeweiligen Satzes verifiziert werden kann (vgl. REHBOCK (1989: 23)). Ist dies der Fall, liegt nach REHBOCK ein ,konstativer“" rhetischer Akt vor. Ist dies nicht der Fall, liegt, wie REHBOCK annimmt, ein ,konstitutiver“ rhetischer Akt vor, der den vom Satz bezeichneten Sachverhalt als Faktum erst schafft (vgl. ibid., 22ff., insbesondere 26f.). Man kann dann sagen, daß die konstativen rhetischen Akte die illokutive Funktion der Mitteilung haben können und die konstitutiven rhetischen Akte die illokutive Funktion der sprachlichen Handlung, die von der EPF beschrieben wird. Auf diese Weise werden die Probleme der Blockierungshypothese vermieden. Ich nenne diese Variante des Performativismus „Konstitutivismus“.

Weshalb auch diese Hypothese von der Zuordnung von Äußerungsbedeutungen und 
illokutiven Funktionen der Äußerungen von Sätzen nicht befriedigend ist, werde ich aus Gründen der Einfachheit der Darstellung später zu zeigen versuchen. Zunächst möchte ich einen Vorschlag zur Konkretisierung des Konstativismus im Hinblick auf die Ableitung der illokutiven Funktion der von der EPF beschriebenen sprachlichen Handlung als illokutive Funktion der EPF-Satz-Äußerung machen. An diesem werde ich dann den Konstitutivismus messen.

\section{Zur Interpretation der illokutiven Funktion explizit performativer Äußerungen im Rahmen des Konstativismus}

Die Ableitung der illokutiven Funktion der von der EPF beschriebenen sprachlichen Handlung als illokutive Funktion der EPF-Satz-Äußerung ist im Rahmen des Konstativismus wie folgt denkbar:

\subsection{Interpretation eines Satzmodus als grammatisch determinierter Bedeutung von Sätzen}

Sätzen der oben genannten für Deklarativsätze typischen Form wird über grammatische Regeln der Laut-Bedeutung-Zuordnung die Interpretation zugeordnet, da $B$ irgendein epistemisches Subjekt den vom Satz bezeichneten relevanten Sachverhalt $\mathbf{r S V}_{s}$ als Faktum in seiner Seinswelt ansieht, wenn der Satz keine diese Einstellung relativierenden Satzadverbiale oder Partikeln enthält (wie z. B. hoffentlich, wahrscheinlich usw.), die die Faktizität des $\mathrm{rSV}_{\mathrm{s}}$ in der Seinswelt des epistemischen Subjekts einschränken. Der Satz drückt also eine epistemische Einstellung eines Einstellungsträgers aus. Dadurch ist diese ein Aspekt seiner grammatisch determinierten Bedeutung (im Sinne von BIERWISCH (1979), (1980)). Gemäß einem pragmatischen Prinzip ist im Defaultfall (d. h. sofern dem nichts entgegensteht) der Einstellungsträger der Sprecher des Satzes. Diese Einstellung zum $\mathrm{rSV}_{\mathrm{s}}$ ist eine Bedingung für Konsistenz im Verhalten des als Einstellungsträger ermittelten Individuums. Jemand, der (2) äußert, kann nicht, ohne formal zu kennzeichnen, daß er sich mit dem von (2) Ausgedrückten geirrt hat, anschließend einen Satz wie (1) (a) äußern. Vgl.

(8) (a) * [Er tut was. (=(2)). Tut er was? (=(1) (a)). $]$

(b) Er tut was. (= (2)). Stopp mal: tut er wirklich was?

Deklarativsätze ohne die Faktizität des von ihnen bezeichneten relevanten Sachverhalts einschränkende Satzadverbiale sind dann Ausdrücke von Urteilen. Sie präsentieren den genannten Sachverhalt als Faktum, indem sie die entsprechende Einstellung eines epistemischen Subjekts zu ihm ausdrücken, das ihn als Faktum festlegt und sich durch die Verwendung des Satzes auf diese Haltung verpflichtet. Die Äußerungen solcher Sätze sind dann Urteilsäußerungen. Die durch die oben illustrierten Formaspekte der Sätze ausgedrückten Typen epistemischer Einstellungen - wie die Einstellung des Urteils (d. h. der Faktizitätsannahme), der Frage (d. h. der Unsicherheit, ob der rSV s $_{\mathrm{s}}$ in Faktum ist oder nicht) usw. nenne ich Satzmodus. ${ }^{16}$

16 Zur Interpretation epistemischer Einstellungen als Aspekt grammatisch determinierter Bedeutung siehe PASCH (1982), (1989), (1990), DOHERTY (1985), (1987), МotsCH/PASCH (1987). 
Exkurs: Gegen die Annahme, daß zur grammatisch determinierten Bedeutung von Sätzen eine epistemische Einstellung eines epistemischen Subjekts gehört, ist Opposition laut geworden (siehe BRANDT/ROSENGREN/ZTMMERMANN (1990)), wenn auch nicht argumentiert worden. Es wurden sog. objektive die Faktizität des $\mathrm{rSV}_{\mathrm{s}}$ tangierende semantische Merkmale als das angesetzt, was unter dem Satzmodus als inhaltlichem Korrelat eines syntaktischen Satztyps zu verstehen ist. Ein offensichtlicher Beweggrund für diese Annahme ist, die illokutive Funktion explizit performativer Äußerungen von EPF-Sätzen ohne die Begriffe des Urteils, der Behauptung und des mit diesen verknüpften Begriffs der Wahrheit ableiten zu können, die ja alle mit der Faktizitätsannahme verbunden sind.

Gegen diese Analyse ist folgendes einzuwenden: Wenn keine Einstellungskomponente als Komponente der grammatisch determinierten Bedeutung der Sätze angenommen wird, bleibt z. B. unklar, wie die entsprechende Annahme eines epistemischen Subjekts zustande kommt, daß es einen Sachverhalt der vom Satz beschriebenen Art gibt, daß ein solcher Sachverhalt ein Faktum ist (vgl. BRANDT/ROSENGREN/ZIMMERMANN (1990: 124)). Eine solche Annahmne ist aber notwendig als eine Prämisse für die Ableitung der illokutiven Funktion „Mitteilung“ der Deklarativsatzäußerungen, denn es ist ja diese Annahme des Sprechers des Satzes und seine Kompetenz und Aufrichtigkeit und nicht etwa die Faktizität des $\mathrm{rSV}_{\mathrm{s}}$ selbst, die einen Adressaten der Satzäußerung zur Realisierung der illokutiven Funktion der Mitteilung, nämlich selbst die Faktizität des $\mathrm{rSV}_{\mathbf{s}}$ anzunehmen, bei der Rezeption von Deklarativsatzäußerungen bewegt. Wäre dies nicht so, bliebe die Effizienz von Lügen unerklärbar.

Dies soll erst einmal genügen. Ich komme auf diesen Punkt noch etwas genauer in Abschnitt 5. zurück. In jedem Fall muß eine Theorie über die Interpretation von Satzäußerungen präzisieren, auf welchem Wege Urteile über Konsistenz bzw. Inkonsistenz im Sprecherverhalten (wie sie durch (8) (a) illustriert wird) zustande kommen. Sie muß angeben, was die Prämissen für solche Urteile sind. Wenn die gen. epistemische Einstellung nicht Bestandteil der grammatisch determinierten Bedeutung der Sätze wäre, d. h. nicht aus der Form der Sätze selbst zu interpretieren wäre (also nicht im sprachlichen Zeichen Satz kodiert wäre), dann müßte ihre Interpretation aus dem Kontext der Satzäußerung abgeleitet werden können, und zwar in jedem Fall. Es bliebe dann die Frage offen, warum Äußerungen von Deklarativsätzen ohne die Faktizität des $\mathrm{rSV}_{\mathrm{s}}$ einschränkende Satzadverbiale nur unter ganz bestimmten Bedingungen nicht als Urteilsäußerungen interpretiert werden können und nicht umgekehrt nur unter bestimmten kontextuellen Bedingungen als Urteile interpretiert werden können, d. h. allgemein gesprochen, woher die Unterscheidbarkeit wörtlicher und nichtwörtlicher Verwendungen der Sätze bezüglich solcher Einstellungen rührt. (Ich denke hier an die Verwendung eines Satzes wie $D u$ rührst dich nicht vom Fleck. als Aufforderung gegenüber seiner Verwendung als Urteilsausdruck etwa in Du siehst, ich strampele mich hier ab, und du rührst dich nicht vom Fleck. mit Vorwurfscharakter.) (Zu einer detaillierteren Kritik an einer Theorie, die Satztypen generell nicht als Mittel des Ausdrucks von Einstellungen annimmt, siehe PASCH (1990).)

\subsection{Interpretation einer Äußerungsbedeutung von Sätzen}

Wie schon angedeutet wurde, ist die grammatisch determinierte Bedeutung eines Ausdrucks nur ein Schema, das im Falle seiner konkreten Anwendung bei einer Verwendung - 
in der Kommunikation: bei einer Äußerung - des Ausdrucks spezifiziert wird bzw. sogar modifiziert werden kann. So ist bei nichtzitierender syntaktisch nichteingebetteter Verwendung eines Deklarativsatzes mit den oben beschriebenen sonstigen Eigenschaften das Subjekt der vom Satzmodus repräsentierten epistemischen Einstellung der Sprecher des Satzes. Macht allgemeines Weltwissen oder der Faktenkontext evident, daß der geäußerte Satz nicht aufrichtig geäußert sein kann, so kommt es zu einer Divergenz zwischen grammatisch determinierter Bedeutung und der Bedeutung der Äußerung des Satzes, die ich hier mit BIERwISCH (1979) „Äußerungsbedeutung“ des Satzes nenne. Dabei sind zwei Fälle zu unterscheiden: a) nur der $\mathrm{rSV}_{\mathrm{s}}$ stimmt nicht mit den evidenten Fakten überein; dies ist der Fall der Ironie; vgl.

(9) Du bist aber rücksichtsvoll!

geäußert bei ganz offensichtlich rücksichtslosem Verhalten des Adressaten; b) die als Satzmodus ausgedrückte epistemische Einstellung ist offensichtlich nicht gegeben; dies ist der Fall bei Rhetorizität; vgl.

(10) Kann man einem nackten Mann in die Tasche greifen?

Hier ist der Satzmodus als Teil der grammatisch determinierten Bedeutung des Satzes der der Frage. Die Äußerungsbedeutung dagegen muß aufgrund von Weltwissen ein Urteil mit dem Inhalt der Negation des $\mathrm{rSV}_{s}$, daß man einem nackten Mann in die Tasche greifen kann, sein.

In diesen Fällen sind die Äußerungsbedeutungen der Sätze „übertragene“ (nichtwörtliche) Bedeutungen. In anderen Fällen können - jenseits der Beseitigung referentieller Vagheiten der grammatisch determinierten Bedeutung durch die Äußerung derselben in einem bestimmten Kontext - grammatisch determinierte Bedeutung und Äußerungsbedeutung übereinstimmen. Dann ist die Äußerungsbedeutung eine ,wörtliche“ Bedeutung. Eine wörtliche Einstellungsinterpretation eines Urteils ist z. B. immer im Falle der explizit performativen Äußerungen von EPF-Sätzen gegeben. ${ }^{17}$ Das liegt daran, daß unabhängig von der Äußerung des Satzes kein Faktum oder Wissensinhalt vorliegt, der das Urteil ad absurdum führen könnte, und daß auch - anders als in (10) - der $\mathrm{rSV}_{s}$ in den betref-

17 ReHrock (1989, 12, Fn. 9) wendet gegen PASCH (1989: 36), wo ich EPF-Sätze als „Urteilsausdrïcke" bezeichne, ein, daß mit einer solchen Analyse rätselhaft bleibe, „warum deren performative Lesart durch assertive Modalpartikeln wie $j a$, doch etc. blockiert wird“. Hierzu ist folgendes zu sagen: Die Aussage, daß ,assertive“ Modalpartikeln die performative (d. h. konstitutive) Lesart generell blockieren, ist zu streng. Wenn doch den Hauptakzent im Satz trägt, ist diese Lesart möglich. Vgl. Ich wollte Sie eigentlich um nichts mehr bitten, aber nun bitte ich Sie (hiermit) doch, daß Sie diesen Antrag prüfen. (Allerdings ist doch dann auch nicht mehr als ,assertive“ Modalpartikel zu bezeichnen, weil es so auch in Entscheidungsfragen vorkommt, wo unbetontes doch ebenfalls nicht verwendet werden kann.) Sicher sind solche Modalpartikelverwendungen ziemlich selten, weil die kontextuellen Bedingungen ihrer Verwendung selten gegeben sein dürften. Generell und uneingeschränkt für alle Fälle, in denen die Modalpartikel nicht den Hauptakzent im Satz trägt, dürfte aber gelten, daB ,assertive“ Modalpartikeln in explizit performativen Äußerungen deshalb nicht zu verwenden sind, weil ihr Gebrauch die Bedeutung des Komplexes aus EPF und ihrem Komplement als Gegenstand einer voraufgehenden Erwartung voraussetzt. Dies ist jedoch in der Regel mit der Konstitution des vom EPF-Satz beschriebenen Sachverhalts als Faktum unverträglich, weil die Konstitution der Satzäußerung als Äußerung mit einer bestimmten illokutiven Funktion per Spezifizierung dieser Funktion durch die EPF in der Regel nicht Gegenstand von Erwartungen ist. Sätze mit solchen Modalpartikeln müssen dann über das Wissen um diesen Zusammenhang als pragmatisch nicht wohlgeformt ausgefiltert werden. 
fenden Sätzen nicht als Tatsache ausgeschlossen ist. So gibt es für die explizit performativen Äußerungen von (4) (a) kein unabhängig von der Äußerung bestehendes Faktum, das das Urteil, daß der Sprecher des Satzes den Adressaten höflichst bittet, den betreffenden Antrag zu prüfen, ad absurdum führt, weil der Sachverhalt, daß der Sprecher das Genannte tut, nicht als Faktum ausgeschlossen ist - weder an sich, noch durch den Äußerungskontext.

Im Hinblick auf die hier behandelten Phänomene sei betont, daß die verwendeten Termini „Urteil"“ und „wahr" bzw. „falsch" keinesfalls mit den Gebrauchsbedingungen der deutschen Wörter „Urteil“, „wahr“ und „falsch“ interpretiert werden dürfen. Um eine solche unerwünschte Identifikation zu vermeiden, hier die inhaltlichen Festlegungen für „Urteil“" und „Wahrheit", wie ich sie als Einheiten meiner Metasprache verstanden wissen möchte:

(i) Ein Urteil ist der von der epistemischen Einstellung $u$ eines Trägers $t$ dieser Einstellung $u$ in einem Einstellungssachverhalt $u(t, s v)$ als Faktum in der Seinswelt von $t$ qualifizierte konzeptuelle (d. h. intensionale) Gegenstand $s v$ von $u$.

(ii) Wahrheit ist eine Eigenschaft eines Urteils im Sinne von (i), die dem Urteil zukommt, wenn es in der Seinswelt des Trägers $t$ der das Urteil als solches qualifizierenden epistemischen Einstellung $u$ einen Sachverhalt $s v^{\prime}$ gibt, der vom konzeptuellen Gegenstand $s v$ der das Urteil stiftenden Einstellung $u$ abgebildet werden kann.

Ein Satz wie (4) (a) kann dann das Urteil ausdrücken, daß der Sprecher den Adressaten höflichst bittet, den betreffenden Antrag wohlwollend zu prüfen, und dieses Urteil kann wahr sein, nämlich dann, wenn der Sprecher den Adressaten um das vom Satz Bezeichnete bittet. Daß der Sprecher auf den Adressaten verbal einwirkt, ist evident. Deshalb kann die Wahrheit dieses Aspekts des Satzes als gegeben gelten und der betreffende Aspekt des durch das Verb ausgedrückten Satzinhaltes mithin als präsupponiert. Was nicht evident ist, ist, daß der Sprecher auf den Adressaten mit einer Bitte einwirkt. Dies ist allein aus dem Satzinhalt zu entnehmen. Hierfür gibt es auch keinen den Satz verifizierenden Tatbestand in der Seinswelt von Sprecher und Adressat, der unabhängig vom Willen des Sprechers bestünde. Wie Behauptungen über Empfindungen des Sprechers, wie

(11) Ich fühle mich nicht gut.

können explizit performative Äußerungen nicht bestritten werden. Anders als Behauptungen von Empfindungen sind die explizit performativen Äußerungen, um die es mir hier geht, die sog. nichtinstitutionellen Sprechakte, bzw., im Sinne von SEARLE (1989: 549), ,linguistic declarations“, aber immer wahr. Dies sieht man z. B. daran, daß eine auf die Äußerung von (4) (a) folgende Äußerung (12) desselben Sprechers, die an denselben Adressaten gerichtet ist wie die Äußerung von (4) (a), falsch ist, und zwar zwangsläufig, wenn ich und Sie und diesen Antrag in (4) (a) und (12) jeweils auf dieselben Entitäten referieren:

(12) Ich habe Sie nicht gebeten, diesen Antrag zu prüfen.

Der Grund dafür ist die Äußerung von (4) (a) und daß das Verb der EPF eine sprachliche Handlung mit der betreffenden illokutiven Funktion bezeichnet, der Satz dem Deklarativsatzmodus angehört und die in Abschnitt 1 genannten zusätzlichen formalen Merkmale aufweist und der Sprecher einer EPF-Satz-Äußerung die Autorität besitzt, seiner Äußerung die illokutive Funktion zuzuschreiben, die er ihr zu geben wünscht. Indem der 
Sprecher so über seine Äußerung das durch den Satz charakterisierte Urteil fällt, schreibt er der Satzäußerung die durch das EPF-Verb bezeichnete illokutive Funktion zu und schafft gleichzeitig das Faktum, durch das die Äußerung wahr gemacht wird. ${ }^{18}$ In diesem Sinn sind explizit performative Äußerungen ,sich selbst verifizierend“, indem sie das Faktum bilden, das ihren Inhalt verifiziert. ${ }^{19}$ Indem der Sprecher des EPF-Satzes seiner Äußerung die vom EPF-Verb bezeichnete illokutive Funktion per Urteil zuschreibt und den EPF-Satz äußert, drückt er diese illokutive Funktion als illokutives Ziel seiner Äußerung aus, „manifestiert“ er die betreffende Äußerungsintention im Sinne von SEARLE (1989: 553). (Daß diese Manifestation nur über den Ausdruck eines Urteils gehen kann, wenn nicht für die EPF-Sätze die oben erwähnte Blockierung der Urteilsableitung angenommen werden soll, bleibt bei SEARLE (1989), der Konstativismus und Performativismus vereinbaren will (siehe ibid., 557) im Dunkel. SEARLE erkennt mit den Performativisten an, daß die illokutive Funktion explizit performativer Äußerungen von EPF-Sätzen die vom EPF-Verb bezeichnete illokutive Funktion ist und mit den Konstativisten, daß diese ÄuBerungen auch einen Wahrheitswert haben und damit Behauptungen (statements) sind. Behauptungen seien sie jedoch nur via Konstitution eines Sprechaktes mit der vom EPF-Verb bezeichneten illokutiven Funktion.)

\section{3. Äußerungsbedeutung und illokutive Funktion, der Äußerung}

Was hat nun der Satzmodus als Aspekt der grammatisch determinierten Bedeutung von Sätzen mit der illokutiven Funktion der Satzäußerungen zu tun? Wie kommen die Äußerungen zu ihrer Zielsetzung in der Kommunikation, die ja mehr sein kann, als daß der Adressat zur Kenntnis nimmt, wie der Sprecher der Satzäußerung zu dem vom Satz bezeichneten relevanten Sachverhalt steht. Als illokutive Funktion von Deklarativsatzäußerungen kann man ja wie gesagt das Ziel annehmen, daß der Adressat von der Faktizität dieses Sachverhalts überzeugt ist und als illokutive Funktion der explizit performativen Äußerungen von EPF-Sätzen kann man ja annehmen, daß ihr Adressat die illokutive Funktion der vom Satz beschriebenen sprachlichen Handlung verwirklicht.

18 Dieser Ansicht sind die meisten Konstativisten, so z. B. ReCANATI (1987: 212) und SEARLE (1989: 538).

Etwas anders sieht BARTSCH (1979: 224) die Frage der Wahrheit explizit performativer Äußerungen. Sie sagt: ,Sobald die Gültigkeitsbedingungen dafür, daß die Äußerung A als Sprechhandlung $H$ gilt, erfüllt sind, ist die Wahrheit jedes den Vollzug der Sprechhandlung $H$ aussagenden Satzes gesichert“. Dabei versteht sie unter „Gültigkeitsbedingungen“ die „Erkennbarkeitsbedingungen " und die „Korrektheitsbedingungen" für Sprechhandlungen (ibid., 219f.). Abgesehen davon, daß die Erkennbarkeitsbedingungen (,normale Bedingungen für sprachliches Produzieren und Wahrnehmen; Interpretationsstufe (1) der Äußerung gemäß den konventionellen Bedeutungen der Ausdrücke"; ibid., 219) für die Bewertung jeglichen sprachlichen Ausdrucks in bezug auf seine inhaltlichen Aspekte erfüllt sein müssen (und von der Erfüllung dieser Bedingungen gehe ich aus, wenn ich von der ,Wahrheit“" explizit performativer Äußerungen spreche), scheint mir die Bindung der Bewertung eines performativ geäußerten EPF-Satzes als wahr an die erfüllung der Korrektheitsbedingungen (,,vorbereitende Bedingungen ..., Ehrlichkeitsbedingungen ..., Intention des Sprechers, daß die Äußerung als Ausdruck seiner Handlungsintention zählt"; ibid.) zu streng. Der Grund ist, daß u. a. die z. B. zwischen (4) (a) und (12) gegebene genannte Beziehung der Unverträglichkeit auch dann gegeben ist, wenn der Sprecher von (4) (a) nicht aufrichtig (ehrlich) war usw.

19 Siehe auch BARTSCH (1979: 224) - mit der in Fußnote 18 genannten Einschränkung -, RECANATI (1987: 212) und SEARLE (1989: 547f.) 
Die Feststellung, daß der Adressat z. B. einer Äußerung von (4) (a) zur Erfüllung der genannten Zielsetzung dieser Äußerung kraft der Äußerung von (4) (a) und nicht etwa zufällig bewegt wird, setzt die Existenz eines ganzen Systems von Regeln der Zuordnung von Zielsetzungen von Äußerungstypen zu Typen von Äußerungsbedeutungen voraus. ${ }^{20}$ So muß für (4) (a) angenommen werden, daß ein Ziel der Äußerung von (4) (a) ist, daß der Adressat von der betreffenden Zielsetzung der Erfüllung der Bitte überzeugt ist. Er muß es als Tatsache ansehen, daß der Sprecher ihn bittet, den genannten Vorschlag wohlwollend zu prüfen. ${ }^{21}$ Dies kann er mit Fug und Recht aufgrund der Äußerungsbedeutung von (4) (a), die ja eine wörtliche ist, weil gegen die grammatisch determinierte Bedeutung von (4) (a) keine Gegenevidenz im Satzinhalt selbst oder im Verwendungskontext des Satzes vorliegt. Dieser Zustand kann über das genannte Regelsystem der Äußerungsbedeutung der Urteilsäußerung von (4) (a) - nämlich daß der Sprecher es als Faktum erachtet, daß er den Adressaten um wohlwollende Prüfung des genannten Vorschlags bittet - als illokutive Funktion (als Zieltyp) der ,Mitteilung“ zugeordnet werden. Die illokutive Funktion der Bitte wird dann über eine andere Regel mit der Äußerung von (4) (a) verknüpft. Im folgenden führe ich noch einmal die in PASCH $(1990,820)$ eingeführten tentativen Formulierungen solcher Regeln an, die ich „Regeln sozialen Verhaltens“ nenne:

(RSV 1) Wenn jemand zu verstehen gibt, daß er ein Problem hat und es lösen will, und wenn man annimmt, daß das zu verstehen Gegebene auch der Fall ist, soll man ihm, wenn nichts dagegen spricht, dabei helfen, wenn er allein zur Lösung nicht in der Lage ist.

(RSV 2) Wenn jemand zu verstehen gibt, daß er etwas wünscht, und wenn man annimmt, daß das zu verstehen Gegebene auch der Fall ist, soll man ihm, wenn nichts dagegen spricht, dabei helfen, den Wunsch zu verwirklichen, wenn er allein dazu nicht in der Lage ist.

(RSV 3) Wenn jemand zu verstehen gibt, daß er etwas als Faktum betrachtet, und wenn man annimmt, daß das zu verstehen Gegebene auch der Fall ist, soll man diese Sicht übernehmen, wenn nichts dagegen spricht. 22

Wenn Bitten Wunschäußerungen sind, dann wird eine Äußerung von (4) (a) als Bedingung in (RSV 3) wirksam und aufgrund dieser Implikation und der Identifizierung der Äußerung als Bitte als Bedingung in (RSV2). Diese Regeln können sich Sprecher für den Ausdruck ihrer kommunikativen Ziele zunutze machen, indem sie Ausdrücke verwenden, die über die Regeln der Grammatik und über Regeln des Zusammenspiels von grammatisch determinierter Bedeutung und Kontextinterpretation zur Interpretation der vom Sprecher intendierten Äußerungsbedeutung des jeweils verwendeten Ausdrucks führen, die als Bedingung in einer der Regeln RSV figuriert. Derjenige, der den betreffenden Ausdruck verwendet, kann, da dann die entsprechende Bedingung aus einer der Regeln RSV erfüllt ist, darauf hoffen, daß die in der betreffenden Regel RSV als gewünschte Konsequenz genannte Folge eintritt, wenn der Adressat der Ausdrucksverwendung kooperativ ist und weitere Bedingungen der Realisierung dieser Konsequenz erfüllt sind. Die von den Regeln RSV 1 bis 3 genannten gewünschten Konsequenzen im Verhalten

${ }^{20}$ Gegen die Identifikation von Bedeutung und illokutiver Funktion siehe u. a. STAMPE (1975), BIERWISCH (1979), (1980), MOTSCH/PASCH (1987), PASCH (1989).

$21 \mathrm{Zu}$ entsprechenden Zielhierarchien siehe MoTsCH/PASCH (1987).

22 Ausfiihrlichere Darlegungen zur Motivation dieser Regelformulierungen finden sich in PASCH (1990).

39 Z. Phonetik (ZPSK) 44 (1991) 5 
von Adressaten kommunikativer Handlungen können auf dieser Grundlage als reguläre illokutive Ziele von Satzäußerungen angesehen werden, die die in den Regeln genannte Bedingung über ihre Bedeutung erfüllen. Wenn dann die Bedingung eine wörtliche Satzäußerungsbedeutung ist, ist die illokutive Funktion der Äußerung auch mit dem Satzmodus korreliert.

\subsection{Zusammenfassung}

Meine Position in der Frage der Ableitung der illokutiven Funktion explizit performativer Äußerungen von EPF-Sätzen sei wie folgt zusammengefaßt:

1. EPF-Sätze haben aufgrund ihres Formtyps eine grammatisch determinierte Bedeutung mit einem Satzmodus, der die Einstellung eines epistemischen Subjekts ausmacht, daß der vom Satz bezeichnete relevante Sachverhalt ein Faktum in der Seinswelt des epistemischen Subjekts ist: der Satzmodus ist damit eine „Urteilseinstellung".

2. Wenn nichts dagegen spricht, ist das epistemische Subjekt der Einstellung in der Äußerungsbedeutung des Satzes der Sprecher des Satzes. Da bei explizit performativen Äußerungen von EPF-Sätzen nichts dagegen spricht, ist das epistemische Subjekt solcher Satzäußerungen der Sprecher des Satzes.

3. Wenn nichts dagegen spricht, ist die Bedeutung der Äußerung eines Satzes jenseits einer referentiellen Spezialisierung identisch mit der grammatisch determinierten Bedeutung des Satzes, sie ist eine ,wörtliche“ Bedeutung. Bei explizit performativen Äußerungen von EPF-Sätzen spricht nichts dagegen, daß ihre Bedeutung eine wörtliche ist. Folglich ist sie die eines Urteils des Sprechers des Satzes.

4. Die Bedeutung der explizit performativen Äußerungen von EPF-Sätzen ist bezüglich des sie in der Seinswelt des Sprechers verifizierenden Sachverhalts konstitutiv, d. h. sie konstituiert diesen als Faktum. Dieses Faktum macht die Äußerungsbedeutungen dieser Sätze wahr, wenn der Sprecher zur Schaffung des betreffenden Faktums autorisiert ist (siehe (4) (a), (b)), oder es macht sie bezüglich eines Wahrheitswertes unentscheidbar, wenn die Autorisierung nicht gegeben ist (z. B. wenn jemand erklärt, er taufe jemanden, etwas, oder verheirate jemanden usw., ohne dazu legitimiert zu sein). Im letztgenannten Fall würde eine Präsuppositionsverletzung vorliegen.

5. Der Bedeutung der explizit performativen Äußerungen von EPF-Sätzen ist über ein System von Regeln sozialen Verhaltens (RSV) das illokutive Ziel der Mitteilung des vom Satz bezeichneten relevanten Sachverhalts an den Adressaten zugeordnet (siehe RSV 3) und via Faktengeltung dieses Sachverhalts die illokutive Funktion, die von der EPF beschrieben wird (für (4) (a) ist dann RSV 2 einschlägig).

6. Der Satzmodus eines Satzes bedingt über die wörtliche Interpretation des Satzes bei seiner Äußerung und über die Regeln RSV ein typisches illokutives Ziel der Satzäußerungen. Weil bei explizit performativen Äußerungen von EPF-Sätzen das letzte an die Form der Sätze gebundene illokutive Ziel der Äußerung die illokutive Funktion der sprachlichen Handlung ist, die von der EPF beschrieben wird, bedingt der Satzmodus in explizit performativen Äußerungen von EPF-Sätzen nur ein der illokutiven Funktion voraufgehendes typisches Ziel und nicht die illokutive Funktion der Äußerung selbst. 


\section{Epilog: Probleme mit dem Konstitutivismus, einer Variante des Performativismus}

Erinnern wir uns: in Abschnitt 3.2. wurde eine Variante des Performativismus behandelt, die die Ableitung der illokutiven Funktion der von der EPF bezeichneten sprachlichen Handlung als illokutive Funktion (fundamentales Ziel) der explizit performativen Äußerungen der EPF-Sätze ohne Blockierung eines mit der Deklarativsatzform assoziierten illokutiven Ziels der Satzäußerung gestatten soll, nämlich eine Variante, die ich „Konstitutivismus“ genannt habe. Mit dieser haben wir eine echte Alternative zu der soeben vorgestellten Variante des Konstativismus, was die Frage der Äußerungsbedeutungen von EPF-Sätzen angeht. Auf der Ebene der Äußerungsbedeutung, zu der die Unterscheidung zwischen wahrheitswertfähigen vs. nichtwahrheitswertfähigen Äußerungen - genauer: Äußerungsinhalten - ja letztlich gehört und damit auch die Unterscheidung von Deklarativsatzäußerungen, die als „Behauptungen“ bezeichnet werden können, von solchen, die nicht so genannt werden können, ist die Frage, welche der beiden Theorien - Konstativismus oder konstitutivistischer Performativismus - die überlegenere ist, nämlich nicht zu entscheiden.

Prüfen wir nun, ob die Entscheidung auf der Ebene der Zuordnung eines illokutiven Ziels - wie des der Mitteilung - zur Bedeutung der explizit performativen Äußerungen von EPF-Sätzen gefällt werden kann. Hierzu gibt es bis jetzt im Rahmen des Konstitutivismus keine Aussagen. Spielen wir also einmal die möglichen Vorstellungen dazu durch. Zu diesem Zweck fasse ich noch einmal die oben angeführte Hypothese darüber zusammen, wie diese Zuordnung im Rahmen des Konstativismus aussehen könnte. Für konstative Äußerungen (z. B. Behauptungen) und konstitutive Äußerungen (z. B. explizit performative Äußerungen von EPF-Sätzen ${ }^{23}$ ), wird einheitlich angenommen, daß ihre Bedeutung darin besteht, daß ein Träger einer epistemischen Einstellung - im allgemeinen der Sprecher des Satzes - den vom Satz bezeichneten Sachverhalt $\mathrm{rSV}_{\mathrm{s}}$ als ein Faktum ansieht. Wenn nichts dagegen spricht, daß der Sprecher ihn als Faktum ansieht, sollte der Äußerungsadressat den $\mathrm{rSV}_{\mathrm{s}}$ ebenfalls als Faktum ansehen, wenn er kooperativ sein will. Wenn der als vom Sprecher des Satzes als Faktum angesehen ausgegebene $\mathrm{rSV}_{\mathrm{s}}$ eine sprachliche Handlung des Sprechers beschreibt, die mit der Äußerung des Satzes selbst identifiziert werden muß, sollte der Adressat der Satzäußerung deren illokutive Funktion verwirklichen. Auf einen kurzen Nenner gebracht besagt dies: der Adressat einer Satzäußerung sollte, wenn er kooperativ sein will, alle mit Aspekten der Bedeutung dieser Äußerung regulär verbindbaren und auf ihn selbst beziehbaren illokutiven Ziele dieser Äußerung, die sich nicht gegenseitig ausschließen, verwirklichen.

Im Rahmen des REHBOCKschen Konstitutivismus (siehe REHBOCK (1989)) wird streng zwischen konstativen und konstitutiven Äußerungsbedeutungen unterschieden. Dabei weist REHBOCK dem Deklarativsatzmodus unabhängig von diesen beiden Arten der Äußerungsbedeutung von Deklarativsätzen folgende Funktion zu: „Der Sachverhalt e [der meinem Begriff des $\mathrm{rSV}_{\mathrm{s}}$ entspricht - R. P.] wird durch den Satzmodus projektiv auf die (jeweilige Diskurs-)Welt bezogen; ihn dort als Fakt aufzusuchen oder neu einzusetzen, ist die Anweisung, die ein Sprecher dem Hörer mit Hilfe des Deklarativsatzmodus vermittelt" (REHBOCK $(1989,6)$ ). Ich muß hier für den Vergleich von Konstativismus und Kon-

23 Konstitutiv können auch explizit performative Äußerungen von Deklarativsätzen ohne EPF sein, wie die Äußerung von Die Sitzung ist eröffnet. (mit fallender Intonation) durch eine zur Sitzungseröffnung befugte Person. 
stitutivismus davon ausgehen, daß - entgegen REHBOCKS Annahme (siehe ibid., 12) - „als Fakt aufsuchen“" und ,als Fakt einsetzen" an die Annahme gebunden sein müssen, daß e vom Sprecher als Faktum angesehen wird. Ohne diese Annahme kann z. B. a) der Deklarativsatzmodus im Falle des ,als-Fakt-Aufsuchens“ nicht mehr vom Entscheidungsinterrogativsatzmodus abgegrenzt werden und b), wie gesagt, die Effizienz von Lügen nicht erklärt werden. 24

In einer Theorie, nach der konstitutive Äußerungen, d. h. Äußerungen, die nach REHBOCK das Faktum ,in die Diskurs-Welt einsetzen", nie gleichzeitig konstativ sein können, kann dann folgende Art der Zuordnung illokutiver Ziele zu Äußerungsbedeutungen angenommen werden:

(i) Für konstative Äußerungen: Wenn der $r S V_{s}$ unabhängig von der Satzäußerung ein Faktum sein kann und der Sprecher des Satzes den rSV $_{\mathbf{s}}$ als Faktum hinstellt und wenn nichts dagegen spricht, daß er ihn als Faktum ansieht, so sollte der Äußerungsadressat den $\mathrm{rSV}_{\mathrm{s}}$ als Faktum ansehen, wenn er kooperativ sein will.

(ii) Für konstitutive Äußerungen (eingeschränkt auf solche von EPF-Sätzen):

Wenn der $\mathrm{rSV}_{\mathrm{s}}$ nicht unabhängig von der Satzäußerung ein Faktum sein kann, sondern vielmehr eine sprachliche Handlung des Sprechers beschreibt, die mit der Äußerung des Satzes selbst identifiziert werden muß, und wenn der Sprecher den $\mathrm{rSV}_{\mathrm{s}}$ als Faktum hinstellt und wenn nichts dagegen spricht, daß der Sprecher den $\mathrm{rSV}_{s}$ als Faktum ansieht ${ }^{25}$, so sollte der Adressat die illokutive Funktion der vom $\mathrm{rSV}_{\mathrm{s}}$ beschriebenen Handlung erfüllen, wenn er kooperativ sein will.

Anders als bei der konstativistischen Behandlung der Zuordnung illokutiver Funktionen von Satzäußerungen zu deren Bedeutungen erscheint die illokutive Interpretation von Äußerungsbedeutungen von EPF-Sätzen im Rahmen des Konstitutivismus formal nicht als Spezialfall der illokutiven Interpretation eines Urteils, obwohl sie das für Urteile zutreffende Merkmal der Erhebung eines Faktizitätsanspruchs für den $\mathrm{rSV}_{\mathrm{s}}$ durch den Sprecher des Satzes enthält. Dieses läuft hier leer, obwohl es gestatten würde, die illokutive Interpretation von EPF-Sätzen als Spezialfall der illokutiven Interpretation von Deklarativsätzen $\mathrm{zu}$ begründen. Dies läuft faktisch auf die Blockierung des illokutiven Ziels hinaus, das Urteilen und damit bestimmten Deklarativsätzen zugeordnet werden kann. Eine solche Blockierungslösung wurde jedoch für die Interpretation von explizit performativen Äußerungen bereits in Abschnitt 3. als ad hoc verworfen. Eine Alternative wäre nur, bei der illokutiven Interpretation von Deklarativsatzäußerungsbedeutungen auf die Disjunktion von konstativen und konstitutiven Äußerungsbedeutungen zu verzichten und diese nur als relevant für die Verifizierung der Deklarativsatzbedeutungen anzusehen und

24 Die Annahme der Faktizität des $\mathrm{rSV}_{\mathrm{S}}$ durch den Sprecher des Satzes bei explizit performativen Äußerungen von EPF-Sätzen geht auch in die Bedingungen für Konsistenz im Verhalten des Sprechers ein. So muß das Verhalten des Sprechers von (4) (a), der später äußert, daß er den Adressaten von (4) (a) nie gebeten habe, den in (4) (a) genannten Antrag zu prüfen (siehe (12)), als inkonsistent bewertet werden.

$25 \mathrm{Da}$ die Komponente, daß der Sprecher den $\mathrm{rSV}_{\mathrm{S}}$ als Faktum ansieht, auch bei explizit performativen Äußerungen von EPF-Sätzen notwendig ist, sieht man daran, daß die EPF allein noch nicht die Konstitutivität der Äußerungsbedeutung und nicht die Performativität der Satzäußerung bezüglich der beschriebenen sprachlichen Handlung ermöglicht. Ein Satz wie Bitte ich Sie hiermit höflichst, diesen Antrag wohlwollend zu prüfen? (mit steigender Intonation geäußert) ist ja wenngleich pragmatisch unsinnig - nach den Regeln der semantischen Interpretation sprachlicher Ausdrücke durchaus bildbar. 
ansonsten eine beiden Typen von Äußerungsbedeutungen gemeinsame illokutive Funktion abzuleiten mit einer Spezialisierung bei den konstitutiven Äußerungen. Dies aber wäre wieder Konstativismus, zu dem der konstitutivistische Performativismus ja gerade als Alternative angeboten wurde.

Aus dieser Sachlage ziehe ich folgende Bilanz: Solange es keine von ad-hoc-Festlegungen freie Alternative zum Konstativismus gibt, bleibt dieser die überzeugendere Lösung des Problems der Zuordnung illokutiver Funktionen zu Äußerungsbedeutungen. Dies umso mehr, als er dieselben Prinzipien bemüht, nach denen auch die sog. indirekten Sprechakte 26 interpretiert werden. (Siehe hierzu ausführlicher PASCH (1990).) 27

\section{Literatur}

AUSTIN, J. L. (1962): How to do things with words, London etc.

BACH, K. and R. M. HARNISH (1979): Linguistic communication and speech acts, Cambridge/Mass.

BARTSCH, R. (1979): Die Rolle von pragmatischen Korrektheitsbedingungen bei der Interpretation von Äußerungen, in: GREWENDORF, G. (Hrsg.), Sprechakttheorie und Semantik, Frankfurt/M., 217-243.

BIERWISCH, M. (1979): Wörtliche Bedeutung - eine pragmatische Gretchenfrage, in: GREWENDORF, G. (Hrsg.), Sprechakttheorie und Semantik, Frankfurt/M., 119-148; des weiteren in LS/ ZISW/A 60, Berlin 1979, 48-80.

- (1980): Semantic structure and illocutionary force, in: SEARLE, J. R., F. KIEFER, and M. BIERwiSCH (eds.), Speech act theory and pragmatics. Dordrecht. (= Synthese Language Library 10: $1-35$ ).

Brandt, M., I. Rosengren und I. ZIMmermann (1990): Satzmodus, Modalität und Performativität, in: ZPSK 43, 1: 120-149.

DOHERTY, M. (1985): Epistemische Bedeutung (= Studia grammatica XXII), Berlin.

- (1987), Epistemic meaning, Berlin, Heidelberg.

FALKenBerG, G. (1989): Explizite Performative sind nicht indirekt, in: Linguistische Berichte 124, 463-469.

-(1990), Explizite Illokutionen, in: ZPSK 43, 4: 500-514.

GREWENDORF, G. (1979a): Haben explizit performative Äußerungen einen Wahrheitswert? in: GREWENDORF, G. (Hrsg.), Sprechakttheorie und Semantik, Frankfurt/M., 175-196.

- (1979b): Explizit performative Äußerungen und Feststellungen, in: GREWENDORF, G. (1979), Sprechakttheorie und Semantik, Frankfurt/M., 197-216.

KaTz, J. J. (1972): Semantic theory, New York, etc.

26 Zur Ableitung der illokutiven Funktion der sog. indirekten Sprechakte siehe SEARLE (1975).

$27 \mathrm{Im}$ Unterschied zu den indirekten Sprechakten ist bei den explizit performativen Äußerungen von EPF-Sätzen die Interpretation der illokutiven Funktion der beschriebenen sprachlichen Handlung als illokutive Funktion der Satzäußerung unabhängig vom Äußerungskontext des Satzes bei Referenz des Satzes auf seine eigene aktuelle Äußerung obligatorisch. Diese ist nicht in höherem Maße nur indirekt an die Satzform gebunden als jegliche illokutive Funktion von Satzäußerungen. Im Gegenteil, sie ist mehr an sie gebunden als die der sog. indirekten Sprechakte, insofern als sie durch den Satz selbst - durch die EPF - beschrieben wird. Sie verlangt nur einen Ableitungsschritt mehr als die Interpretation einer illokutiven Funktion einer Satzäußerung, die via wörtliche Äußerungsbedeutung des Satzes allein mit dem Satzmodus korreliert ist. Dies jedoch ist etwas ganz anderes als die "Indirektheit" von Satzäußerungen, die traditionell als Fehlen der Verankerung der Interpretation der illokutiven Funktion der Satzäußerung in der Form des Satzes verstanden wird. (Siehe MOTSCH/PASCH (1987: 65).) Deswegen kann den Konstativismus der hier vorgestellten Art der wiederholt vorgebrachte Vorwurf (siehe hierzu zuletzt FALKENBERG (1989)), der Konstativismus behandle explizit performative Äußerungen von EPF-Sätzen gegen alle Intuition als indirekte Sprechakte, nicht treffen. Explizit performative Äußerungen von EPFSätzen sind auch in dieser Variante des Konstativismus keine indirekten Sprechakte. 
LEwIS, D. (1972): General semantics, in: DAVIDSON, D. and G. HARMAN (eds.), Semantics of natural language, Dordrecht, 169-218.

LIEDTKE, F. (1990): Performativität, Sprechhandlung, Wahrheit, in: ZPSK 43, 4: 515-532.

PASCH, R. (1982):Illokutionäre Kraft von Äußerungen und semantischer Satztyp, in: LS/ZISW/A 91, II, Berlin, 112-155.

- (1985): Typen von Einstellungsbekundungen, in: ZfGerm 6-1, 53-63.

- (1989): Uberlegungen zum Begriff des „Satzmodus“, in: LS/ZISW/A 193, Berlin, 1-88; Kurzfassung in: ZPSK 43 (1990) 1: 92-110

- (1990): Satzmodus als Grundlage illokutiver Funktionen: Kritische Fragen zu BRANDT/ROSENGREN/ZIMMERMANN: Satzmodus, Modalität und Performativität, in: ZPSK 43 (1990) 1 , 120-149, in: ZPSK 43, 6: 810-822.

Posner, R. (1980): Theorie des Kommentierens. Eine Grundlagenstudie zur Semantik und Pragmatik (= Linguistische Forschungen 9), 2., verbess. und erweit. Aufl., Wiesbaden.

RECANATI, F. (1980): Some remarks on explicit performatives, indirect speech acts, locutionary meaning and truth value, in: SEARLE, J. R., F. KIEFER, and M. BIERWISCH (eds.), Speech act theory and pragmatics, Dordrecht, etc., 205-220. (= Synthese Language Library 10).

- (1987): Meaning and force. The pragmatics of performative utterances, New York, etc.

REHBOCK, H. (1989): Deklarativsatzmodus und pragmatische Interpretation, in: S \& P 15, Lund.

RolF, E. (1986): Eine handlungsanalytische Kritik der Sprechakttheorie, in: Linguistische Berichte $106,470-483$.

- (1987): Über den Deklarativsatzmodus, in: MeIBAUER, J. (Hrsg.), Satzmodus zwischen Grammatik und Pragmatik. Referate anläßlich der 8. Jahrestagung der Deutschen Gesellschaft für Sprachwissenschaft, Heidelberg 1986, Tübingen (=Linguistische Arbeiten 180: 190-206).

ROSENGREN, I. (1984): Die Einstellungsbekundung im Sprachsystem und in der Grammatik, in: STICKEL, G. (Hrsg.), Pragmatik in der Grammatik. Jahrbuch 1983 des Instituts für deutsche Sprache, Düsseldorf, 152-174.

- (1985): Die Beziehungen zwischen Sprachhandlungssystem und Sprachsystem am Beispiel der Einstellungsbekundung, in: ZfGerm 6-3, 322-337.

- (1988): Die Beziehung zwischen Satztyp und Illokutionstyp aus einer modularen Sicht, in: LS/ZISW/A 185, 113-138.

SEARLE, J. R. (1969): Speech acts, Cambridge.

- (1975): Indirect speech acts, in: Cole, P, and J. L. MORGAN (eds.), Syntax and Semantics, vol. 3: Speech acts, New York, 59-82.

- (1989): How performatives work, in: Linguistics \& Philosophy 12-4, 535-558.

STAMPE, D. W. (1975): Meaning and truth in the theory of speech acts, in: COLE, P. and J. L. MORGAN (eds.), Syntax and Semantics, vol. 3: Speech acts, New York, 1-39.

Dr. ReNATE PASCH, Zentralinstitut für Sprachwissenschaft, Berlin 